University of Nebraska - Lincoln

DigitalCommons@University of Nebraska - Lincoln

1976

\title{
Comparison of Experimental and Theoretical Electron Ejection Cross Sections in Helium by Proton Impact from 5 to $100 \mathrm{keV}$
}

\author{
M. Eugene Rudd \\ University of Nebraska - Lincoln, erudd@unl.edu \\ D. H. Madison \\ Drake University, Des Moines, lowa
}

Follow this and additional works at: https://digitalcommons.unl.edu/physicsrudd

Part of the Physics Commons

Rudd, M. Eugene and Madison, D. H., "Comparison of Experimental and Theoretical Electron Ejection Cross Sections in Helium by Proton Impact from 5 to 100 keV" (1976). M. Eugene Rudd Publications. 50. https://digitalcommons.unl.edu/physicsrudd/50

This Article is brought to you for free and open access by the Research Papers in Physics and Astronomy at DigitalCommons@University of Nebraska - Lincoln. It has been accepted for inclusion in M. Eugene Rudd Publications by an authorized administrator of DigitalCommons@University of Nebraska - Lincoln. 
Published in Physical Review A 14, 128 - 136 (1976)

\title{
Comparison of experimental and theoretical electron ejection cross sections in helium by proton impact from 5 to $100 \mathrm{keV}$
}

\author{
M. E. Rudd * \\ Behlen Laboratory of Physics, University of Nebraska, Lincoln, Nebraska 68588 \\ D. H. Madison ${ }^{\dagger}$ \\ Department of Physics, Drake University, Des Moines, Iowa 50311
}

Received 3 March 1976

Absolute values of doubly differential cross sections are obtained for electron production in helium by 5- to $100-\mathrm{keV}$ proton impact. The measured values along with earlier data at higher energies are compared with theoretical plane-wave Born-approximation functions obtained from a Hartree-Fock potential. Not only is good agreement obtained at high impact energies (above $300 \mathrm{keV}$ ), but fairly good agreement is found at low energies (below about $20 \mathrm{keV}$ ). For electrons ejected in the backward hemisphere, unexpectedly poor agreement is noted at intermediate energies where the measured cross sections dip to $50 \%$ of the calculated values in some cases. Singly differential cross sections integrated over all angles agree quite well with the theoretical calculations over all ranges of parameters studied. Singly differential cross sections integrated over all electron energies tend to be more isotropic in angle as the proton energy is decreased from 100 to $5 \mathrm{keV}$. Total cross sections for electron production are also obtained which compare reasonably well with those of Solov'ev et al. but which are higher than those of de Heer et al. It is shown that the contribution to the cross section by the mechanism of charge transfer to the continuum decreases as the impact energy decreases, contrary to the prediction of Salin's treatment.

(C1976 The American Physical Society

URL: http://link.aps.org/doi/10.1103/PhysRevA.14.128

DOI: $10.1103 /$ PhysRevA.14.128

${ }^{*}$ Work supported by the National Science Foundation under Grant No. MPS73-04777 A01.

${ }^{\dagger}$ Work supported by the Research Corporation. 


\title{
Comparison of experimental and theoretical electron ejection cross sections in helium by proton impact from 5 to $100 \mathrm{keV}$
}

\author{
M. E. Rudd* \\ Behlen Laboratory of Physics, University of Nebraska, Lincoln, Nebraska 68588 \\ D. H. Madison $\dagger$ \\ Department of Physics, Drake University, Des Moines, Iowa 50311 \\ (Received 3 March 1976)
}

\begin{abstract}
Absolute values of doubly differential cross sections are obtained for electron production in helium by $5-$ to $100-\mathrm{keV}$ proton impact. The measured values along with earlier data at higher energies are compared with theoretical plane-wave Born-approximation functions obtained from a Hartree-Fock potential. Not only is good agreement obtained at high impact energies (above $300 \mathrm{keV}$ ), but fairly good agreement is found at low energies (below about $20 \mathrm{keV})$. For electrons ejected in the backward hemisphere, unexpectedly poor agreement is noted at intermediate energies where the measured cross sections dip to $50 \%$ of the calculated values in some cases. Singly differential cross sections integrated over all angles agree quite well with the theoretical calculations over all ranges of parameters studied. Singly differential cross sections integrated over all electron energies tend to be more isotropic in angle as the proton energy is decreased from 100 to $5 \mathrm{keV}$. Total cross sections for electron procluction are also obtained which compare reasonably well with those of Solov'ev et al. but which are higher than those of de Heer $\boldsymbol{e t}$ al. It is shown that the contribution to the cross section by the mechanism of charge transfer to the continuum decreases as the impact energy decreases, contrary to the prediction of Salin's treatment.
\end{abstract}

\section{INTRODUCTION}

In an earlier study, ${ }^{1}$ it was seen that experimental angular distributions of electrons ejected from helium by $100-300-\mathrm{keV}$ protons agreed reasonably well with calculations using the scaled hydrogenic Born approximation at intermediate angles, but large discrepancies were observed for small and large angles. Subsequently it was shown ${ }^{2-5}$ that a mechanism not included in the Born formulation, namely, charge transfer into continuum states, was responsible for the increased cross sections at small angles. Although the available theoretical treatments of this mechanism do not yield close quantitative agreement with experiment, there is general agreement that this mechanism is responsible for the forward peak in the angular distribution.

More recently, the large-angle discrepancy was largely corrected by replacing the scaled hydrogenic wave functions with more realistic ones. ${ }^{6,7}$ With these modifications, data from $100 \mathrm{keV}$ to $5 \mathrm{MeV}$ now seem to be reasonably well accounted for in the present theory, and there is little reason to expect that any large discrepancy will arise at still higher impact energies. However, the situation at lower energies is less clear, since substantial errors seem to be present in the theoretical descriptions of even the total electron ejection cross sections below $100 \mathrm{keV} .{ }^{8}$ Therefore we have taken data on the angular and energy dis- tribution of electrons ejected from helium by protons over the energy range $5-100 \mathrm{keV}$ for comparison with the best Born calculations to determine the limit of applicability of this approximation to this process. The experimental data presented here are also of basic interest to a number of applied fields.

In the work of Crooks and Rudd ${ }^{4}$ it was found that the effect of the mechanism of charge transfer to the continuum apparently decreased as the proton energy was increased from 100 to $300 \mathrm{keV}$. A further decrease was found at higher energies by Manson et al., ${ }^{7}$ and at $5 \mathrm{MeV}$ the cross section due to this mechanism became negligible compared to that due to direct ionization. However, no investigation of this type has been made at lower energies. The present data supply some information on this point as well.

\section{EXPERIMENTAL METHOD}

A magnetically analyzed proton beam entered a double-walled collision chamber similar to one described earlier. ${ }^{8}$ The beam was collimated by two apertures, the one nearest the scattering center being $1.5 \mathrm{~mm}$ in diameter. The combination restricted the diameter of the beam at the collision center to $2 \mathrm{~mm}$. A deep ( $3 \mathrm{~mm}$ diameter by $25 \mathrm{~mm}$ ) Faraday cup collected the beam after it traversed the gas cell. Electrons from a length $4.5 \mathrm{~mm} / \sin \theta$ at the center were allowed to enter 
a $127^{\circ}$ electrostatic analyzer, where $\theta$ is the angle of ejection measured from the beam direction. The angular acceptance of the slit system was $\pm 1.5^{\circ}$ and the effective energy width of the analyzer $^{9}$ was $4.42 \%$. The effective solid angle of the detection system as seen from the scattering center was $5.36 \times 10^{-4} \mathrm{sr}$. No preacceleration was used, thus insuring that the acceptance geometry could be accurately calculated. Electrons were accelerated by $82 \mathrm{~V}$ after analysis before striking the first dynode of an 18-stage electron multiplier (EMI 9642/3B). A fine wire screen shielded the analyzer from the clectric field in the detector.

Magnetic fields were nulled by three pairs of Helmholtz coils to less than about $5 \mathrm{mG}$. Stray electric fields were minimized by carefully polishing and cleaning the brass surfaces in the target chamber, electron pipe, and analyzer. Since it was suspected that a small field could leak from a biased Faraday cup, the cup was used without bias. A deep cup was used to prevent secondary electrons from escaping. Target gas was supplied in cylinders at $99.995 \%$ purity and brought to the gas cell through an all-metal regulator and gas line controlled by a needle valve. Target pressures, measured by a Baratron capacitance manometer, ranged between 0.18 and $1 \mathrm{mTorr}$. Since the manometer head was heated, a $4 \%$ correction was made for the effect of thermal transpiration. In addition, account was taken of the nonzero Baratron reference pressure obtained from the outer part of the scattering chamber. For helium it was found by direct measurement that the ratio of inner- to outer-chamber pressures was 46 , and this figure was used to make the correction.

A correction was also made for a small error caused by neutralization of the beam between the analyzing magnet and the Faraday cup. This was done using a method described by Rudd and Macek. ${ }^{10}$ Similarly, a correction was made for electrons absorbed between the collision center and detector. At the pressures used, the neutralization correction ranged from $0 \%$ to $4 \%$, while the absorption correction never exceeded $8 \%$ and was generally much smaller.

Dead-time losses in the counting system were corrected by using the equation $R^{*}=R /\left(1-R T_{D}\right)$ given by Evans. ${ }^{11} R^{*}$. is the true and $R$ is the measured counting rate, and $T_{D}$ is the dead time. The dead time was essentially that of the discriminator and was found to be $1.1 \mu \mathrm{sec}$ by the use of a pulse-pair generator and oscilloscope. This time agreed with oscilloscope measurements made using random pulses produced by an electron gun directed at the detector. An attempt was also made to measure the dead time by recording elec- tron counts from the primary beam for constant charge and varying currents. This yielded a dead time of $2.5 \mu \mathrm{sec}$. The discrepancy is not understood, but the lower value was chosen for the calculations. The counting rates were kept low enough that the greatest value for this correction was $7 \%$.

A system of movable apertures employed by Cacak and Jorgensen ${ }^{12}$ was used to measure the efficiency of the electron detector. An electronemitting filament was placed at the scattering center and current from it through a large aperture was compared to the count rate with a small aperture moved into place. Measurements of the sizes of the apertures with a traveling microscope gave a ratio of areas which agreed with that obtained previously ${ }^{12}$ by measuring ratios of currents. Efficiencies were measured as a function of electron energy and were found to fall approximately linearly from 0.80 at $30 \mathrm{eV}$ to 0.70 at $300 \mathrm{eV}$. The proper value of efficiency at each energy was used, taking into account the $82-\mathrm{V}$ acceleration to the first dynode. The uncertainty in the efficiency measurement was $5 \%$.

Electron counts were taken at 17 electron energies between 1.5 and $300 \mathrm{eV}$ with helium in the target chamber. A background run was then taken with the target gas off. The proton energy was subsequently changed and the procedure repeated until runs at all nine proton energies were completed before changing to the next angle. Total counts and background counts were stored in a multiscaler and read out on paper tape for computer processing.

The proton energy was taken to be the sum of the accelerator terminal potential and the potential on the extraction electrode of the $\mathrm{rf}$ ion source. The former was measured with a voltage divider (calibrated to within $\frac{1}{4} \%$ ) and a differential voltmeter. The extraction potential varied between 400 and $3600 \mathrm{~V}$, with the lower values being used at the lower proton energies. This procedure yielded the same proton energy as direct measurements of the beam energy made with a high-resolution cylindrical electrostatic analyzer.

Over most of the range of parameters reported here the uncertainty in the doubly differential cross section is $20 \%$. Below $20 \mathrm{eV}$ the effect of residual magnetic fields and stray electric fields of unknown origin causes the uncertainty to rise to $50 \%$ at $10 \mathrm{eV}$ and greater at lower electron energies. At a sufficiently high electron energy the count rate decreased to the point where statistical uncertainties became important. This is most serious at angles above $70^{\circ}$ and for low proton energies, where the beam current available was small. At $100 \mathrm{keV}$, where the present data can be 
compared with earlier data, the discrepancy is generally less than $10 \%$ with the data of Rudd, Sautter, and Bailey ${ }^{1}$ and less than $20 \%$ with the data of Rudd and Jorgensen. ${ }^{8}$

\section{THEORY}

In the plane-wave Born approximation, the triply differential cross section for ionization of an atom by an incident particle of charge $Z_{p}$ is given by

$$
d \sigma_{f i}=\frac{2 Z_{p}^{2}}{v^{2}}\left|F_{f i}\left(q, \phi_{p}, E_{e}, \Omega_{e}\right)\right|^{2} \frac{d \ln q^{2}}{q^{2}} d \phi_{p} d E_{e} d \Omega_{e},
$$

where $v$ is the relative velocity between the incident particle and the atom, $q$ is the momentum transferred to the atom, $\phi_{p}$ is the azimuthal scattering angle of the projectile, and $E_{e}$ and $\Omega_{e}$ are the energy and angle of observation for the ejected electron. The form factor is given by

$$
F_{f i}=\left\langle U_{f}^{(-)}(\overrightarrow{\mathrm{k}}, \overrightarrow{\mathrm{r}})\left|e^{i \overrightarrow{\mathrm{d}} \cdot \overrightarrow{\mathrm{r}}}\right| U_{i}(\overrightarrow{\mathrm{r}})\right\rangle .
$$

The single-particle wave function for the initial bound electron is $U_{i}$; for the final continuum electron of momentum $\overrightarrow{\mathrm{k}}$ it is $U_{f^{*}}$. The differences in the many calculations labeled "Born approximation" lie primarily in the choice of wave functions $U_{i}$ and $U_{f}$. The customary practice has been to use hydrogenlike wave functions for $U_{f}$ with various types of wave functions for $U_{i}$ ranging from hydrogenlike to very good correlated helium-atom wave functions. However, the cross sections obtained from these approaches do not agree well with experiment. To get good agreement with experiment, it is necessary to use realistic wave functions for $U_{f}$ as well as $U_{i}$. For this calculation, $U_{i}$ and $U_{f}$ were calculated as bound and con- tinuum eigenfunctions of a central potential $V(r)$ obtained from Hartree-Fock bound-state wave functions for helium,

$$
V(r)=-4 / r+(4 / r) Y_{0}(1 s, 1 s / r),
$$

where

$$
Y_{0}(1 s, 1 s / r) \equiv \int_{0}^{r} P_{1 s}^{2}\left(r^{\prime}\right) d r^{\prime}+\int_{r}^{\infty} P_{1 s}^{2}\left(r^{\prime}\right) \frac{r}{r^{\prime}} d r^{\prime} .
$$

The bound-state radial wave function $P_{1 s}(r)$ was obtained from the self-consistent Hartree-Fock program of Froese-Fischer. ${ }^{13}$ The potential (3) is asymptotically zero, as it should be for the incident channel. In the exit channel, the atomic potential should be asymptotically Coulombic for a net charge of +1 . Madison and Shelton ${ }^{15}$ have shown that orthogonality considerations require that both the incident and exit channel wave functions be calculated using the same potential, and that best agreement with experiment is obtained when incident channel potentials are used. Consequently, for this calculation the incident channel wave functions were used in the calculation of $V(r)$. However, $V(r)$ was modified to be Coulombic in the asymptotic region by setting it equal to $-2 / r$ when that value was reached. This procedure both guarantees the orthogonality requirement and gives the continuum wave function the proper asymptotic behavior while maintaining the essential features of the incident channel potential.

If the continuum wave function is expanded in partial waves, it can be shown after some angular momentum algebra that the cross section integrated over the proton azimuthal scattering angle, summed over final magnetic states, and averaged over initial magnetic states is given by ${ }^{6}$

$$
\begin{array}{r}
\sigma(E, \theta, q)=\frac{1}{2 \pi k} \sum_{l \lambda} i^{l-\lambda}(2 \lambda+1)(2 l+1)^{1 / 2} C\left(l_{0} \lambda l ; 000\right) f_{l \lambda l_{0}}^{k q} \sum_{l^{\prime} \lambda^{\prime}} i^{l^{\prime}-\lambda^{\prime}}\left(2 \lambda^{\prime}+1\right)\left(2 l^{\prime}+1\right)^{1 / 2} C\left(l_{0} \lambda^{\prime} l^{\prime} ; 000\right) f_{l^{\prime} \lambda^{\prime} l_{0}}^{k q *} \\
\times \sum_{l_{3}} C\left(l l^{\prime} l_{3} ; 000\right) C\left(\lambda^{\prime} \lambda l_{3} ; 000\right) W\left(\lambda l_{0} l_{3} l^{\prime} ; l \lambda^{\prime}\right) P_{l_{3}}(\beta) P_{l_{3}}(\theta),
\end{array}
$$

where $\beta$ is the angle between $\vec{q}$ and the initial momentum of the projectile $\overrightarrow{\mathrm{K}}_{i}, \theta$ is the angle between $\overrightarrow{\mathrm{k}}$ and $\overrightarrow{\mathrm{K}}_{i}, C\left(l_{1} l_{2} l_{3} ; m_{1} m_{2} m_{3}\right)$ is a Clebsch-Gordan coefficient, $W\left(j_{1} j_{2} j_{3} j_{4} ; j_{5} j_{6}\right)$ is a Racah coefficient, and $P_{l}$ is an ordinary Legendre polynomial. The radial form factor is given by

$$
f_{l \lambda l_{0}}^{k q}=\left\langle r^{-1} \chi_{l}(k r)\left|j_{\lambda}(q r)\right| r^{-1} u_{l_{0}}(r)\right\rangle .
$$

Here $u_{l}$ and $\chi_{l}$ are the $l$ th radial components of the bound and continuum eigenfunctions of $V(r)$ and $j_{l}$ is a spherical Bessel function. Doubly differential cross sections for the ejected electron may be obtained from Eq. (4) by numerically integrating over $q$.

\section{RESULTS AND CONCLUSIONS}

In Figs. 1 and 2 are shown the angular distributions of 30 - and 100-eV electrons ejected by protons of various energies. The experimental cross 


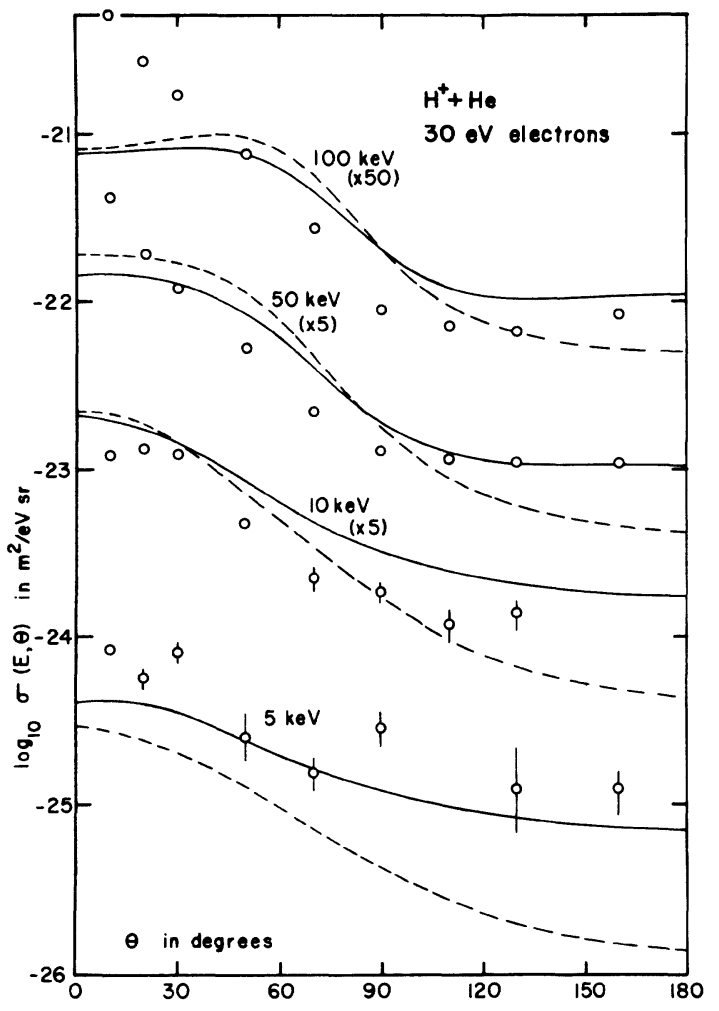

FIG. 1. Angular distributions of $30-\mathrm{eV}$ electrons ejected from helium by protons of various energies. Circles are present experimental points; full line is the Born approximation with Hartree-Fock wave functions; dashed line is the scaled Born approximation with hydrogenic wave functions.

sections are compared with scaled hydrogenic Born-approximation calculations and with Born calculations using wave functions obtained from a Hartree-Fock potential, as described. Since neither calculation contains the mechanism of charge transfer to the continuum, the cross sections in the forward direction are underestimated, particularly where the electron and proton velocities are approximately equal. In the backward direction, the calculations obtained using HartreeFock wave functions are in much better agreement with experiment than the scaled hydrogenic calculations. This improvement is similar to that noted earlier ${ }^{6,7}$ at higher energies. It is remarkable that the agreement is reasonably good even at $5 \mathrm{keV}$, the lowest energy measured so far. This is also seen in Figs. 3 and 4, where cross sections are plotted as a function of proton energy for selected angles and electron energies. One notes no large systematic discrepancy which increases at low energies. This is surprising, since a 5$\mathrm{keV}$ proton has a velocity equal to that of a $2.8-\mathrm{eV}$

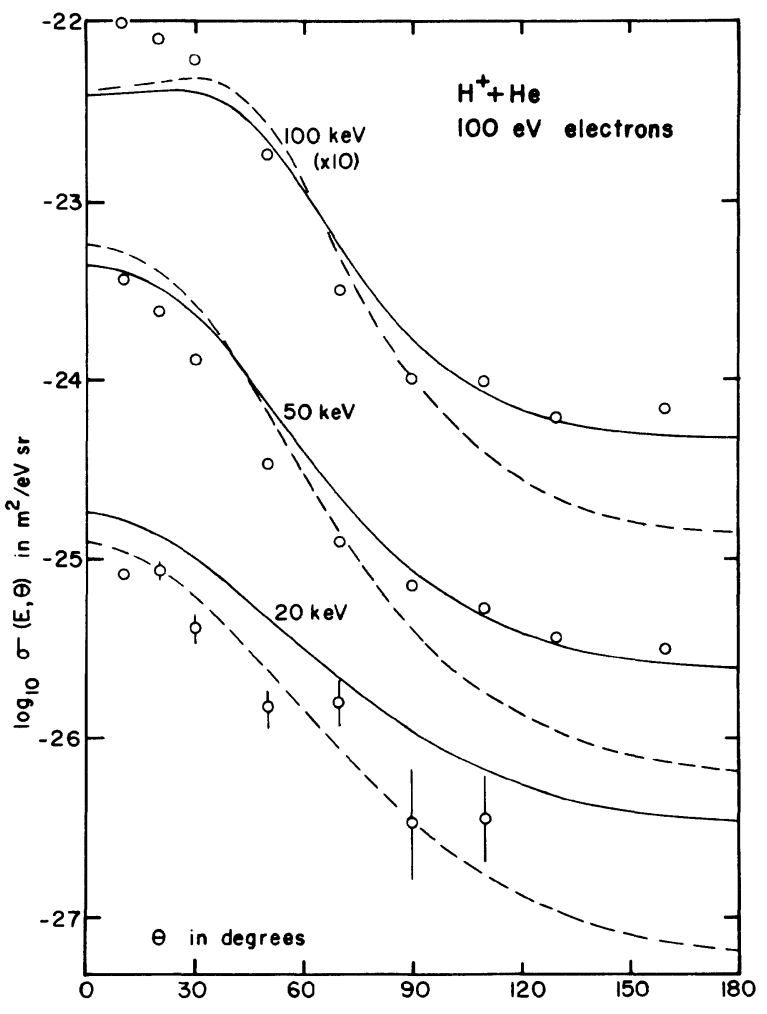

FIG. 2. Same as Fig. 1, but for 100-eV electrons.

electron and the Born approximation is usually thought to hold well only at impact velocities large compared to orbital velocities. Figure 5 shows that the overall agreement at $10 \mathrm{keV}$ is rather good.

Figures 3 and 4 reveal an unexpected behavior for low electron energies and large angles. The agreement with theory is very good at high proton energies, as expected, and reasonably good at low proton energies, as mentioned above. However, at intermediate impact energies there is a distinct dip in the data, producing a departure from theory which reaches a factor of 2 and more. For $90^{\circ}$ scattering, this dip extends from 20 to $300 \mathrm{keV}$ for $10-\mathrm{eV}$ electrons, but its width decreases with increasing electron ejection energy and has virtually disappeared at $100 \mathrm{eV}$. No ready explanation appears for this behavior, but it is perhaps significant that the low-energy end of the dip appears where the proton velocity equals that of the ejected electron. The equal-velocity points are indicated by small vertical arrows next to each curve. For angles in the back hemisphere the upper end of the dip is more or less constant at about $300 \mathrm{keV}$, independent of electron energy and angle. For angles below $90^{\circ}$, however, the 


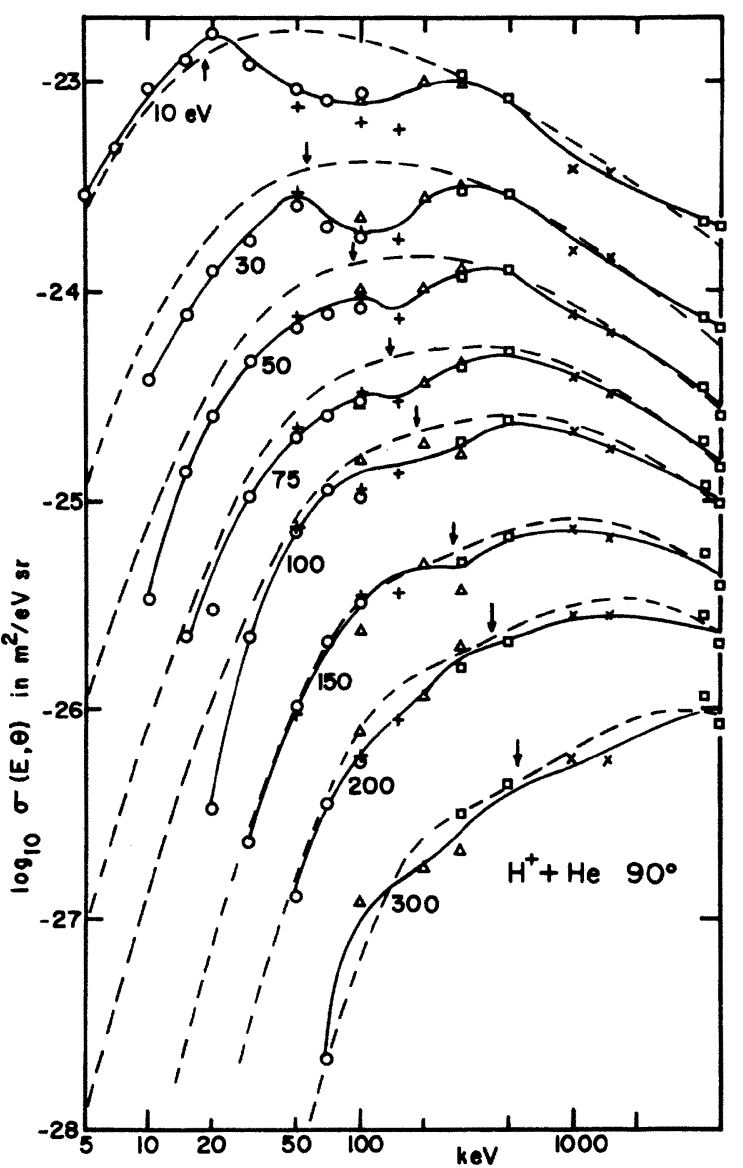

FIG. 3. Doubly differential cross sections for electron ejection at $90^{\circ}$ for various electron energies as a function of proton energy. $O$, present data; $\Delta$, data of Rudd, Sautter, and Bailey (Ref. 1); +, data of Rudd and Jorgensen (Ref. 8); $\times$, data of Toburen (Ref. 7); $\square$, data of Stolterfoht (Ref. 7); dashed line, Born HartreeFock calculations; solid line, composite experimental results. Arrows indicate protons with same velocity as ejected electrons.

dips seem to shift toward lower proton energies. Similar graphs have also been plotted for hydrogen, nitrogen, and argon, but the dips for these gases are much less pronounced than for helium.

In Fig. 6 singly differential cross sections integrated over all angles, as calculated from the equation

$$
\sigma(E)=2 \pi \int_{0}^{\pi} \sigma(E, \theta) \sin \theta d \theta
$$

are shown in comparison with the Born HartreeFock calculations. The agreement is good at 100 $\mathrm{keV}$, worsens at intermediate energies, but improves again at lower impact energies contrary

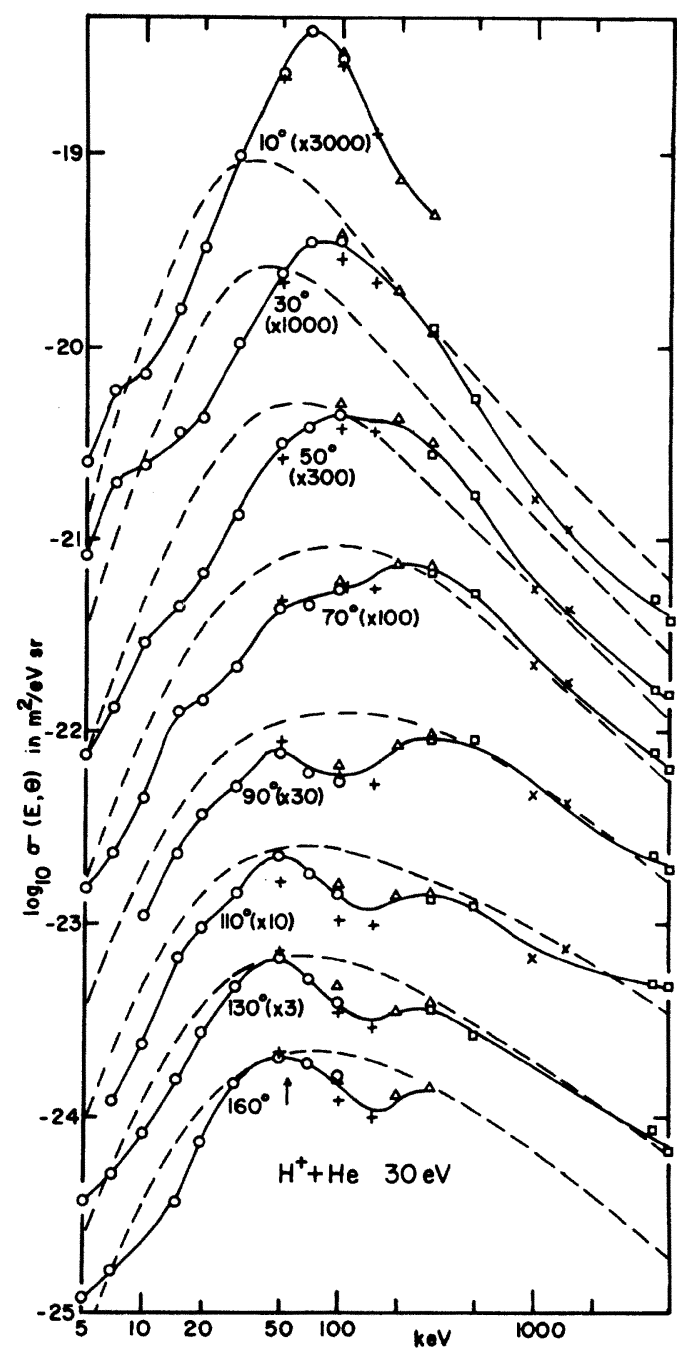

FIG. 4. Doubly differential cross sections for ejection of $30-\mathrm{eV}$ electrons at various angles as a function of proton energy. Legend same as Fig. 3.

to Born-approximation expectations. It should also be noted that the binary-encounter approximation $^{14}$ yields results for these singly differential cross sections which are very close to the Born results. Figure 7 shows the same data plotted in a different way.

Singly differential cross sections integrated over electron energies by the relation

$$
\sigma(\theta)=\int_{0}^{\infty} \sigma(E, \theta) d E
$$

are presented in Fig. 8. Data from other investigators are again added to give a more complete picture. The angular distributions are isotropic 


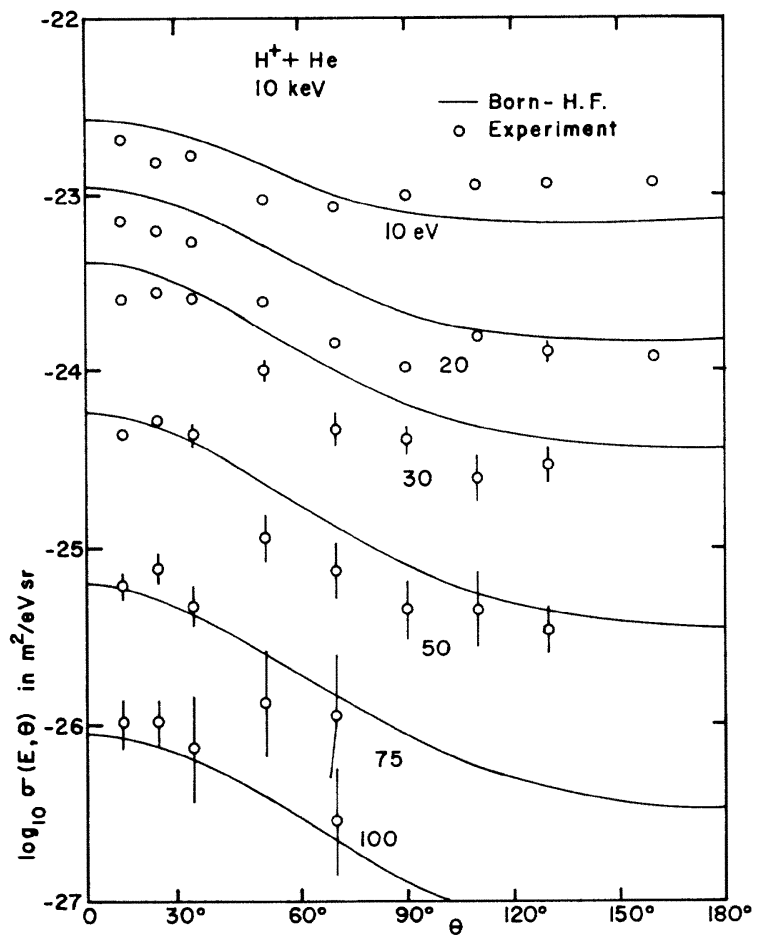

FIG. 5. Angular distributions of electrons ejected at various energies from helium by $10-\mathrm{keV}$ protons. Circles are present experimental points, full line is the theoretical Born Hartree-Fock calculation.

within a factor of 2 at $5 \mathrm{keV}$, but as the impact energy is increased a large peaking in the forward direction develops until there is a factor of $30-35$ between the cross sections at the forward $\left(10^{\circ}\right)$ and backward directions at $100 \mathrm{keV}$. At still higher energies, the distribution becomes more isotropic again and the maximum in the curves moves to larger angles, reaching nearly $90^{\circ}$ at $5 \mathrm{MeV}$.

Another quantity of interest which can be found from the data is the average electron energy, defined by

$$
\bar{E}(\theta)=\int_{0}^{\infty} E \sigma(E, \theta) d E / \int_{0}^{\infty} \sigma(E, \theta) d E
$$

This is plotted in Fig. 9 as a function of angle. The maximum in all curves below $1 \mathrm{MeV}$ seems to come at about $20^{\circ}$ but shifts markedly to larger angles at the highest energies. Curiously, it does not appear that the average energy ever exceeds $110 \mathrm{eV}$ regardless of impact energy or angle.

Figure 10 displays the total electron ejection

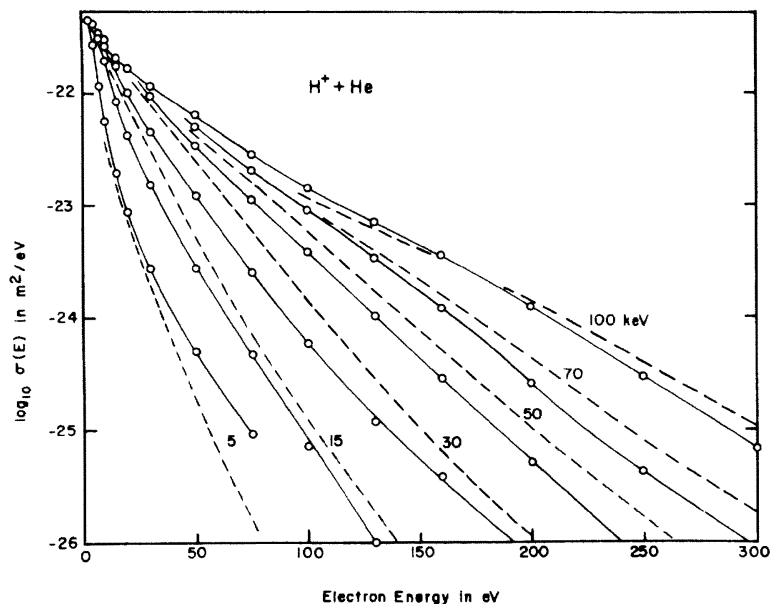

FIG. 6. Energy distribution of electrons integrated over all angles. Full line and circles, present experiment; dashed line, Born Hartree-Fock calculations.

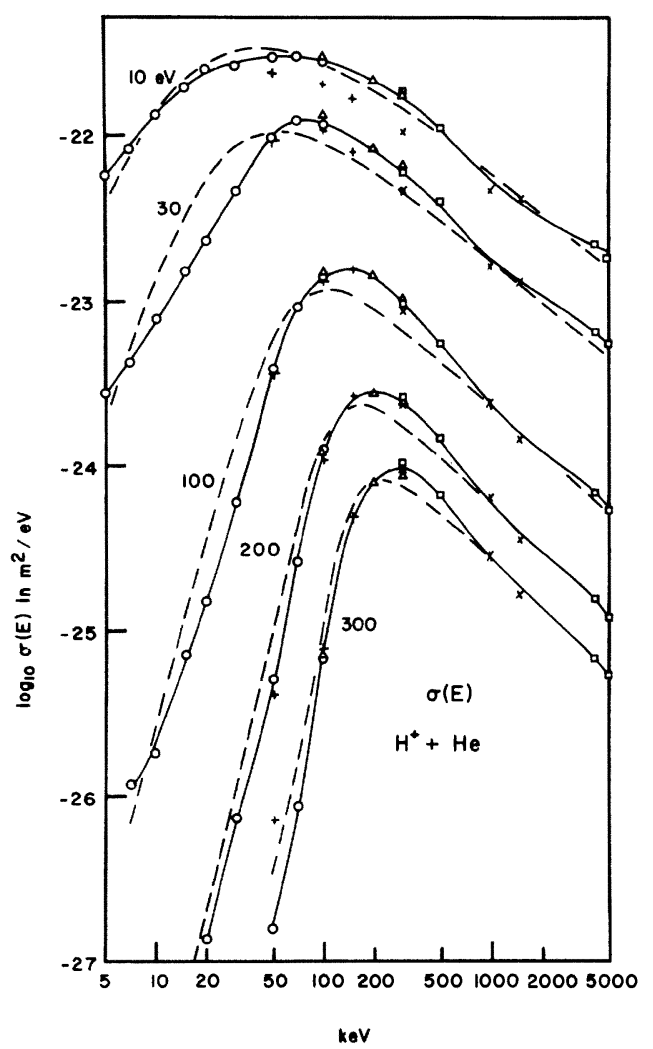

FIG. 7. Cross sections integrated over all angles vs proton energy for various ejected electron energies. Legend as in Fig. 3. 


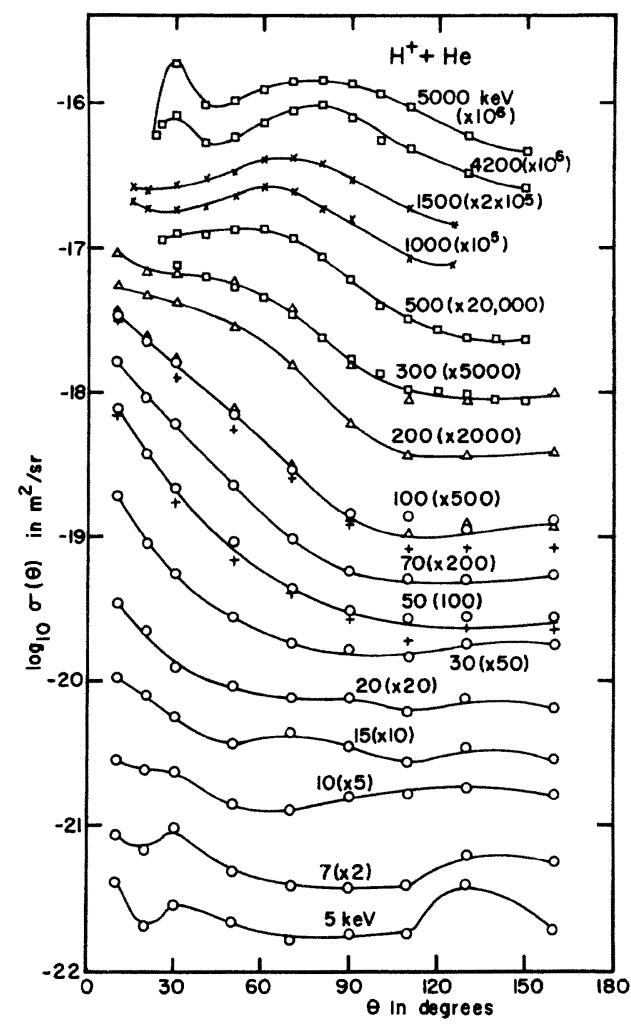

FIG. 8. Angular distributions of electrons of all energies for various proton impact energies. Legend same as Fig. 3.

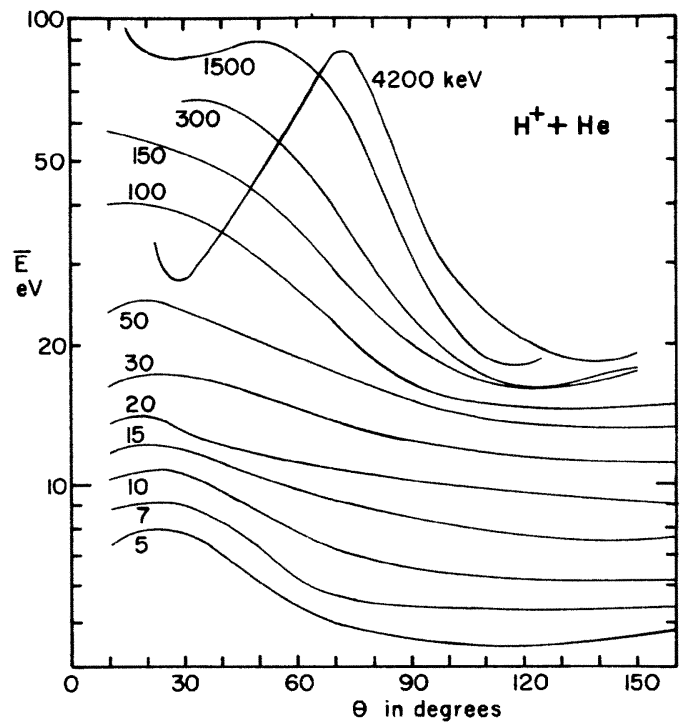

FIG. 9. Average energy of electrons ejected in various directions for various impact energies. Data above $150 \mathrm{keV}$ are from Ref. 7.

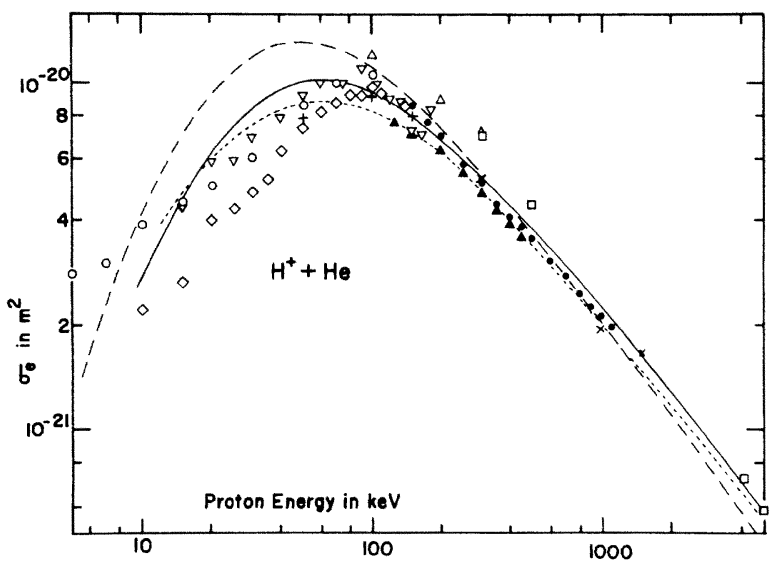

FIG. 10. Total cross sections for production of electrons in helium by protons. $\bullet$, Hooper et al. (Ref. 16); $\nabla$, Solov'ev et al. (Ref. 17); $\Delta$, Gilbody and Lee (Ref. 18); $\diamond$, de Heer et al. (Ref. 19); legend for other data points as in Fig. 3; solid line, Bell and Kingston (Ref. 20); dashed line, scaled from Bates and Griffing (Ref. 21); dotted line, Mapleton (Ref. 22).

cross section given by

$$
\sigma_{e}=2 \pi \int_{0}^{\infty} \int_{0}^{\pi} \sigma(E, \theta) \sin \theta d \theta d E .
$$

The two integrations for the present data were performed in both orders and averaged. Differences between the two procedures were very small. Also shown in Fig. 10 are the results of a number of other experiments and of three different theoretical calculations. From 10 to $100 \mathrm{keV}$ the scaled hydrogenic Born calculations of Bates and Griffing clearly overestimate the data. While the other two calculations are closer in absolute value, the shape at low energies is not much improved.

In Fig. 11 we attempt to show the variation in importance of the mechanism of charge transfer to the continuum as the proton impact energy is varied. According to Salin's treatment, ${ }^{2}$ the maximum value of the ratio of his cross section to the unmodified Born-approximation results (labeled $|N|^{2}$ in Ref. 2) comes at the electron velocity $k=v \cos \theta$, where $v$ is the proton velocity. Therefore for each proton energy we plot the doubly differential cross section at the electron energy $\left(E_{p} / 1836\right) \cos ^{2} \theta$ divided by the cross section at the same energy calculated by the Born Hartree-Fock method. Since the Born calculation does not include this mechanism of electron production, the increase in this ratio over unity can largely be attributed to electrons produced through this mechanism. A similar graph was plotted for high energies by Manson et al., ${ }^{7}$ but for $k=v$. Our 


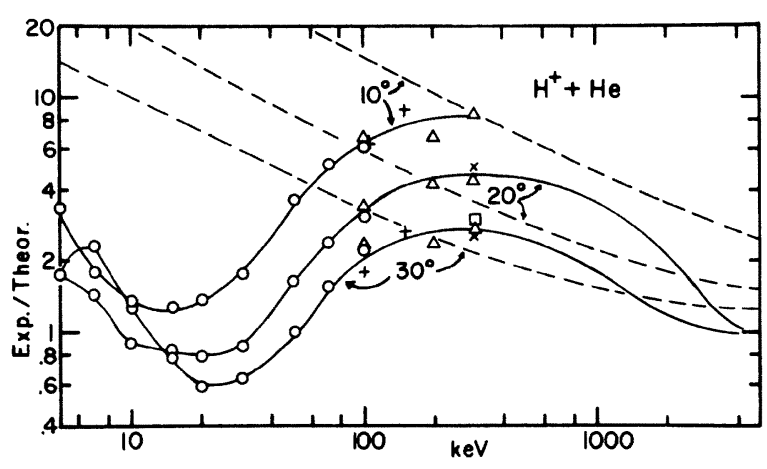

FIG. 11. Ratio of experimental to theoretical (Born Hartree-Fock) doubly differential cross sections calculated for matching velocity protons and electrons (see text). Legend for data points same as Fig. 3. Graph is extended above $300 \mathrm{keV}$ using data from Ref. 7. Dotted line is $|N|^{2}$ factor of Salin. ${ }^{2}$

present graph has been extended to high energies using values taken from their graph. While Salin's $|N|^{2}$ values show a rough correspondence with experiment at high energies, the ratio decreases below $300 \mathrm{keV}$, in contrast to theory, and is close to unity at $15 \mathrm{keV}$. Below that energy there is evidently an upward trend, but the data are not as reliable in that region, and in addition the theoretical values were obtained by extrapolation. One may conclude, however, that the mechanism of charge transfer to the continuum becomes unimportant below about $20 \mathrm{keV}$.

It is also interesting to examine the behavior of the cross sections in the region where the charge exchange effect is evidently unimportant. From Fig. 11, it can be seen that for $30^{\circ}$ scattering, this effect becomes small around $50 \mathrm{keV}$, and that for lower energies the theoretical cross sections at the velocity matching region become larger than the experiment. In Fig. 12, the ratios of theoretical to experimental doubly differential cross sections at $30^{\circ}$ scattering are plotted as a function of electron energy for incident proton energies of 10,20 , and $50 \mathrm{keV}$. In each case, the ratio has a maximum value and appears to approach unity on either side. The arrows on each curve indicate velocity matching energies. The solid arrow on each curve corresponds to $k=v$, and the open arrow corresponds to $k=v \cos \theta$. (The open arrow is off the scale for the $10-\mathrm{keV}$ curve.) The value at the open arrow is the inverse of the corresponding point on Fig. 11. In each case, the peak in the curves occurs for electron velocities con-

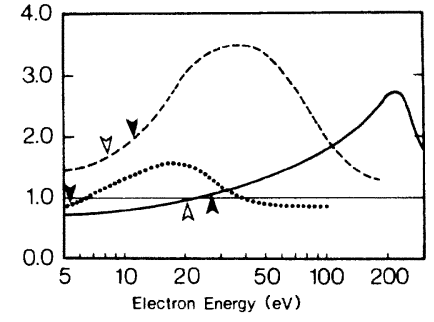

FIG. 12. Ratio of theoretical (Born Hartree-Fock) to experimental doubly differential cross sections for $30^{\circ}$ scattering as a function of ejected electron energy. Dotted line, $10 \mathrm{keV}$; dashed line, $20 \mathrm{keV}$; full line, $50 \mathrm{keV}$.

siderably larger than the proton velocity. These maxima indicate the presence of some energy-dependent mechanism reducing the cross section below the theoretical predictions. The good agreement between experiment and theory at the velocity matching regions is curious, since the Born approximation is expected to give the worst results here due to the large final-state electronproton interaction which is neglected. (This logic implicitly assumes that the electron and proton leave the collision at approximately the same time.)

In conclusion, we have examined doubly differential cross sections for $5-100-\mathrm{keV}$ proton-impact ionization of helium. Comparison of experimental and theoretical results revealed no systematic breakdown of the Born approximation for decreasing proton energies. In fact, good agreement between experiment and theory was observed for the lowest proton energies considered. This is quite surprising when one considers that a 5$\mathrm{keV}$ proton is moving about the same speed as a 2.8-eV electron and that the Born approximation is supposed to be a high-energy approximation. A systematic examination of the comparison between experiment and theory indicates unexplained mechanisms which can have a significant effect on the experimental data.

\section{ACKNOWLEDGMENTS}

We wish to thank C. A. Blocker, G. L. Webster, K. M. Bauer, and E. P. Rudd for their help on the experimental project. We are grateful to L. H. Toburen and N. Stolterfoht for providing their experimental data in tabular form and to S. T. Manson for helpful discussions. 
*Work supported by the National Science Foundation under Grant No. MPS73-04777 A01.

†Work supported by the Research Corporation.

${ }^{1}$ M. E. Rudd, C. A. Sautter, and C. L. Bailey, Phys. Rev. 151, 20 (1966).

${ }^{2}$ A. Salin, J. Phys. B $\underline{2}, 631$ (1969).

${ }^{3}$ J. Macek, Phys. Rev. A 1, 235 (1970).

${ }^{4}$ G. B. Crooks and M. E. Rudd, Phys. Rev. Lett. $\underline{25}$, 1599 (1970).

${ }^{5}$ Y. B. Band, Phys. Rev. A $\underline{8}, 2857$ (1973); $\underline{8}, 243$ (1973).

${ }^{6}$ D. H. Madison, Phys. Rev. A 8, 2449 (1973).

${ }^{7}$ Steven T. Manson, L. H. Toburen, D. H. Madison, and N. Stolterfoht, Phys. Rev. A 12, 60 (1975).

${ }^{8}$ M. E. Rudd and T. Jorgensen, Jr., Phys. Rev. 131, 666 (1963)

${ }^{9}$ C. E. Kuyatt, in Methods in Experimental Physics, edited by B. Bederson and W. L. Fite (Academic, New York, 1968), Vol. 7A, p. 1.

${ }^{10}$ M. E. Rudd and J. H. Macek, Case Stud. At. Phys. $\underline{3}$, 47 (1972).

${ }^{11}$ R. D. Evans, The Atomic Nucleus (McGraw-Hill, New York, 1955), p. 785.

${ }^{12}$ R. K. Cacak and T. Jorgensen, Jr., Phys. Rev. A $\underline{2}$, 1322 (1970).
${ }^{13}$ C. Froese Fischer, Comput. Phys. Commun. $\underline{4}, 107$ (1972).

${ }^{14}$ M. E. Rudd and D. Gregoire, Physics of the One- and Two-Electron Atoms, edited by F. Bopp and H. Kleinpoppen (North-Holland, Amsterdam, 1969), p. 795.

${ }^{15}$ D. H. Madison and W. N. Shelton, Phys. Rev. A $\underline{7}, 499$ (1973).

${ }^{16} \mathrm{~J}$. W. Hooper, Ph.D. thesis (Georgia Institute of Technology, 1961) (unpublished); J. W. Hooper, D. S. Harmer, D. W. Martin, and E. W. McDaniel, Phys. Rev. 125, 2000 (1962).

${ }^{17}$ E. S. Solov'ev, R. N. Il'in, V. A. Oparin, and N. V. Fedorenko, Zh. Eksp. Teor. Fiz. 42, 659 (1962) [Sov. Phys.-JETP 15, 459 (1962)].

${ }^{18}$ H. B. Gilbody and A. R. Lee, Proc. R. Soc. A 274, 365 (1963).

${ }^{19}$ F. J. de Heer, J. Schutten, and H. Moustafa, Physica (Utr.) 32,1766 (1966).

${ }^{20}$ K. L. Bell and A. E. Kingston, J. Phys. B $\underline{2}, 653$ (1969).

${ }^{21}$ D. R. Bates and G. Griffing, Proc. Phys. Soc. Lond. A 66, 961 (1953).

${ }^{22}$ R. A. Mapleton, Phys. Rev. 109, 1166 (1958). 